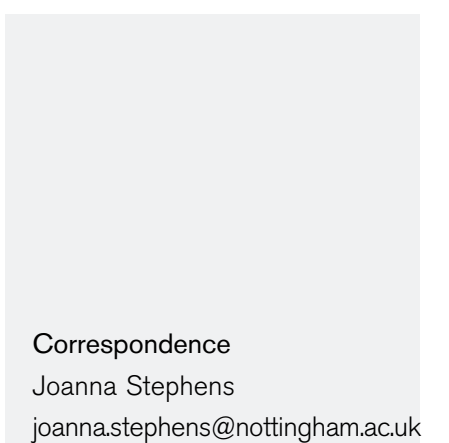

Received 16 September 2014 Accepted 3 May 2015

\section{Streptococcus thermophilus bacteraemia in a patient with transient bowel ischaemia secondary to polycythaemia}

\author{
Joanna Stephens ${ }^{1}$ and David Turner ${ }^{1,2}$ \\ ${ }^{1}$ Nottingham University Hospitals NHS Trust, Derby Road, Nottingham NG7 2UH, UK \\ ${ }^{2}$ Centre for Biomolecular Sciences, School of Life Sciences, University of Nottingham, Nottingham \\ NG7 2RD, UK
}

\begin{abstract}
Introduction: The ability of Streptococcus thermophilus to convert lactose into lactic acid has long been utilized by the dairy industry. A seemingly low-pathogenicity organism, to the best of our knowledge there have been no previously published reports linking the consumption of foodstuffs to bacteraemia caused by this bacterium.
\end{abstract}
Case presentation: Here we present a case of a regular consumer of Activia yoghurt who developed $S$. thermophilus bacteraemia, probably due to transient bowel ischaemia secondary to polycythaemia.

Conclusion: The isolation of this bacterium from blood cultures may indicate underlying large bowel pathology and should not necessarily be dismissed as contamination. Keywords: bacteraemia; polycythaemia; Streptococcus thermophilus.

\section{Introduction}

Infection secondary to the consumption of probiotic products is a safety concern, but despite the widespread consumption of these products, reports of infections by probiotic organisms are extremely rare. A previous literature review identified 15 reports of infection associated with the consumption of products containing probiotic organisms, none of which mention Streptococcus thermophilus as a cause.

S. thermophilus (previously known as Streptococcus salivarius subsp. thermophilus) is a Gram-positive, $\alpha$-haemolytic bacterium that is ubiquitous in the dairy industry. Classified as a 'lactic acid bacterium', the organism's ability to convert lactose to lactic acid is harnessed in the manufacture of a number of dairy products such as yoghurts and cheeses. It is regarded as a low-pathogenicity organism, as genomic studies have shown that common streptococcal virulence genes are either absent or switched off in this species (Prajapati et al., 2013). It also has 'Qualified Presumption of Safety' status in the European Union, as there is a long history of safe use in the food industry (Iyer et al., 2010). In fact, it is thought that over $1 \times 10^{21}$ live $S$. thermophilus bacteria are consumed every year around the world (Hols et al., 2005).

The current case, an immunocompetent patient with intercurrent transient bowel ischaemia on a background of secondary polycythaemia, represents the first reported case, to the best of our knowledge, of bacteraemia due to S. thermophilus.

\section{Case report}

An 86-year-old lady presented to the Accident and Emergency Department at Nottingham University Hospitals NHS Trust, with a $4 \mathrm{~h}$ history of right-sided lower abdominal pain and anorexia. The pain was colicky in nature and rated as $8 / 10$ on a pain scale. She had no nausea or vomiting, no urinary tract symptoms and her bowels were functioning normally. Her past medical history included early-onset Alzheimer's disease, polycythaemia secondary to ischaemic heart disease (JAC2 mutation negative), atrial fibrillation, hypertension and peripheral vascular disease. Her drug history included digoxin, bisoprolol, enalapril, co-amilofruse, simvastatin, aspirin and amitriptyline. She lived with her son who was her full-time carer. On examination, her temperature was $38.4{ }^{\circ} \mathrm{C}$, heart rate was 88 beats $\min ^{-1}$ and blood pressure was $135 / 84 \mathrm{mmHg}$. On chest auscultation, breath and heart sounds were normal and no murmurs were audible. Her abdomen was diffusely tender with more severe tenderness and guarding in the right iliac fossa. The differential diagnosis included acute appendicitis, diverticulitis and acute bowel ischaemia.

\section{Investigations}

An erect chest radiograph was performed, which showed no air under the diaphragm. An abdominal radiograph was also undertaken, which showed no signs of bowel obstruction. A full blood count showed a raised white cell count of $12.3 \times 10^{9} 1^{-1}$ with a neutrophilia $\left(9.8 \times 10^{9}\right.$ 
$\left.\mathrm{l}^{-1}\right)$ and haemoglobin of $179 \mathrm{~g}^{-1}$. Her lactate level was $1.8 \mathrm{mmol} \mathrm{l}^{-1}$. Renal and liver function tests were unremarkable. A urine dipstick was negative for nitrites and leukocytes. Two sets of blood cultures were obtained and incubated in a BACTEC FX blood-culture system (BD Diagnostic Systems). She was referred to the surgical team and empirical therapy with piperacillin/tazobactam $4.5 \mathrm{~g}$ every $8 \mathrm{~h}$ intravenously was initiated. The following day, she underwent a computed tomography scan of the abdomen, which showed no radiological features of acute appendicitis or diverticulitis. Taken together, the clinical presentation and investigations suggested that bowel ischaemia was the most likely diagnosis. Her abdominal pain and fever resolved completely with conservative management, and she was discharged later the same day with a 5 -day course of co-amoxiclav 250/125 mg every 8 h orally.

The day after discharge, both the aerobic and anaerobic bottles from one of the two sets of blood cultures taken on admission signalled as positive (after $16 \mathrm{~h}$ of incubation) and Gram-stained smears from both bottles showed Gram-positive cocci in chains (Fig. 1). After $24 \mathrm{~h}$ of incubation in $5 \% \mathrm{CO}_{2}$, small, grey, $\alpha$-haemolytic colonies were visible on 5\% horse blood agar (Fig. 2). A catalase test reaction and bile aesculin were negative, and the organism was presumptively identified as belonging to the 'viridans streptococci' group. The organism was further identified as S. thermophilus using a VITEK system (bioMérieux), and this was subsequently confirmed by $16 \mathrm{~S}$ rRNA gene sequencing (Molecular Identification Services Unit; Public Health England, UK). The organism was sensitive to amoxicillin using British Society for Antimicrobial Chemotherapy antimicrobial susceptibility methodology. Upon further questioning of the patient's son, it became apparent that she regularly consumed Activia yoghurt (The Danone Company).

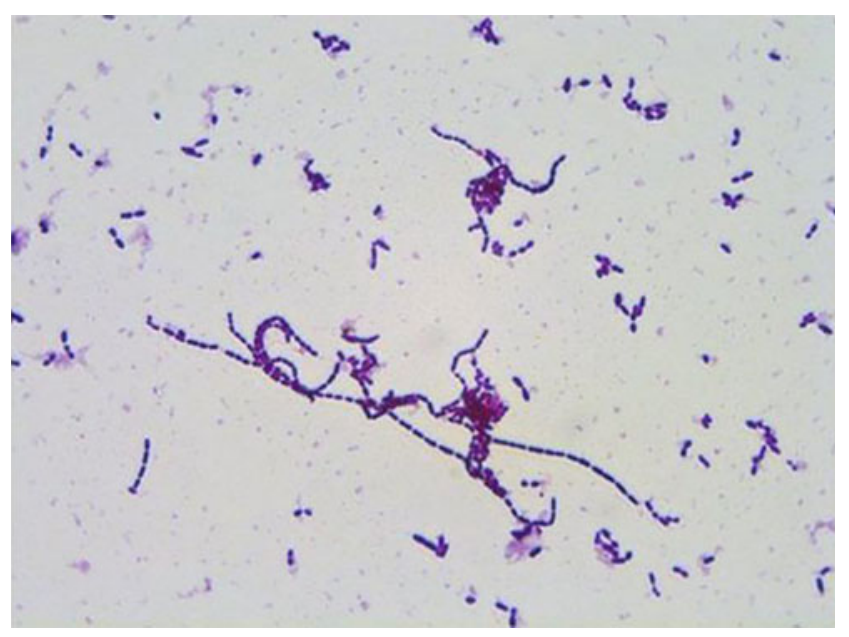

Fig. 1. Gram stain of $S$. thermophilus from broth culture after $16 \mathrm{~h}$ incubation.

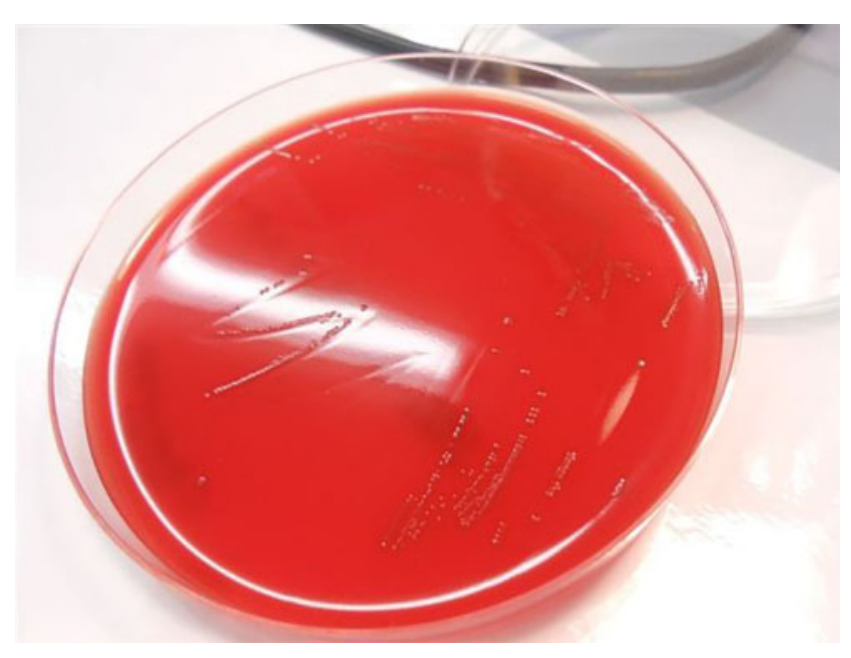

Fig. 2. Colonies of $S$. thermophilus on $5 \%$ horse blood agar after $24 \mathrm{~h}$ incubation in $5 \% \mathrm{CO}_{2}$.

\section{Outcome and follow-up}

On review, following the identification of $S$. thermophilus in the blood culture, the patient remained asymptomatic. The 5-day course of co-amoxiclav was completed. No further blood cultures were obtained. On review 6 months following discharge, she had had no further episodes of abdominal pain or fever.

\section{Discussion}

For centuries, people have knowingly ingested dairy products containing bacteria due to perceived health benefits. A probiotic is defined as 'live micro-organisms that when ingested in large enough quantities confer a health benefit on the host' (Fuller, 1991). The most commonly used bacteria in probiotics are species from the genera Streptococcus, Lactobacillus and Bifidobacterium. The probiotic used by the patient in this case was the yoghurt Activia (The Danone Company), which contains S. thermophilus, Lactobacillus bulgaris and Bifidobacterium lactis. A number of claims have been made concerning the benefits of probiotic products to the consumer's health, from promises of improved 'gastrointestinal health' to the lowering of serum cholesterol levels. Over recent years, the study of probiotics and their effect on health has been substantial. Athough there has been much research, there is little evidence to show that probiotics do in fact confer significant health benefits. However, one area in which probiotics do appear to be beneficial is in the treatment of diarrhoea. A recent meta-analysis looked at the effect of probiotics on antibiotic-associated diarrhoea and found that they did have a protective effect (D'Souza et al., 2002). The effect of probiotics on acute infectious diarrhoea has also been subjected to meta-analysis, which also demonstrated a beneficial effect (Sazawal et al., 2006). 
An obvious issue with ingestion of live micro-organisms is the assurance of safety. Probiotics should not cause infection in the consumer. This may be more of a risk in certain situations such as this case (transient ischaemia allowing translocation), those with damaged intestinal integrity secondary to chemotherapy or radiotherapy, infants with an immature intestinal barrier and those with an immunocompromised immune system. There have been a number of case reports of patients developing bacteraemia, which were thought to be secondary to probiotic consumption (Boyle et al., 2006). To the best of our knowledge, there have been no previous reports of bacteraemia with $S$. thermophilus associated with probiotic consumption. As an $\alpha$-haemolytic streptococcus, $S$. thermophilus in a single blood culture may easily be dismissed as a mere contaminant (and may not be identified to the species level in all cases), particularly if the patient is not displaying overt signs of sepsis. There are also reports of difficulty confirming the identification of $S$. thermophilus by $16 \mathrm{~S}$ rRNA gene sequencing alone (Wilck et al., 2001) or matrix-assisted laser desorption/ionization time-of-flight mass spectrometry fingerprinting (Christner et al., 2010). We hypothesize that consuming Activia yoghurt on a regular basis caused $S$. thermophilus to become part of this patient's large intestinal microbiota. Transient bowel ischaemia, which was likely to be secondary to polycythaemia, probably permitted the organism to translocate and enter the bloodstream.

When one considers the sheer volume of probiotic products consumed around the world, and the possibility of large intestinal colonization, transient bacteraemia with various probiotic organisms is likely to occur on occasion due to intercurrent pathology of the large intestine. We would like to emphasize that this case does not signify safety issues with probiotic products containing $S$. thermophilus; indeed, colonization of the large intestine with $S$. thermophilus (and other probiotic organisms) might protect against translocation-related bacteraemia due to more pathogenic organisms (Sahin et al., 2012). Clinical microbiologists should, however, be aware of this organism and consider the possibility of large bowel pathology rather than contamination if it is identified in bloodculture specimens.

\section{Acknowledgements}

Informed consent to publish this case report was obtained from the patient's son on 30 July 2014, as the patient lacked capacity to consent due to Alzheimer's disease. The authors have no interests to declare.

\section{References}

Boyle, R. J., Robins-Browne, R. M. \& Tang, M. L. K. (2006). Probiotic use in clinical practice: what are the risks? Am J Clin Nutr 83, 1263-1264, quiz 1446-1447.

Christner, M., Rohde, H., Wolters, M., Sobottka, I., Wegscheider, K. \& Aepfelbacher, M. (2010). Rapid identification of bacteria from positive blood culture bottles by use of matrix-assisted laser desorptionionization time of flight mass spectrometry fingerprinting. J Clin Microbiol 48, 1584-1591.

D'Souza, A. L., Rajkumar, C., Cooke, J. \& Bulpitt, C. J. (2002). Probiotics in prevention of antibiotic associated diarrhoea: metaanalysis. BMJ 324, 1361.

Fuller, R. (1991). Probiotics in human medicine. Gut 32, 439-442.

Hols, P., Hancy, F., Fontaine, L., Grossiord, B., Prozzi, D., LeblondBourget, N., Decaris, B., Bolotin, A., Delorme, C. \& other authors (2005). New insights in the molecular biology and physiology of Streptococcus thermophilus revealed by comparative genomics. FEMS Microbiol Rev 29, 435-463.

Iyer, R., Tomar, S. K., Maheswari, T. U. \& Singh, R. (2010). Streptococcus thermophilus strains: multifunctional lactic acid bacteria. Int Dairy J 20, 133-141.

Prajapati, J. B., Nathani, N. M., Patel, A. K., Senan, S. \& Joshi, C. G. (2013). Genomic analysis of dairy starter culture Streptococcus thermophilus MTCC 5461. J Microbiol Biotechnol 23, 459-466.

Sahin, I., Acar, S., Ozaydın, I., Ozaydın, C., Calışkan, E., Yavuz, M. T. \& Iskender, A. (2012). [Investigation of the effects of probiotic bacteria on bacterial translocation that developed during diagnostic laparoscopy: an experimental study]. Mikrobiyol Bul 46, 660-670, (in Turkish).

Sazawal, S., Hiremath, G., Dhingra, U., Malik, P., Deb, S. \& Black, R. E. (2006). Efficacy of probiotics in prevention of acute diarrhoea: a meta-analysis of masked, randomised, placebo-controlled trials. Lancet Infect Dis 6, 374-382.

Wilck, M. B., Wu, Y., Howe, J. G., Crouch, J. Y. \& Edberg, S. C. (2001). Endocarditis caused by culture-negative organisms visible by Brown and Brenn staining: utility of PCR and DNA sequencing for diagnosis. J Clin Microbiol 39, 2025-2027. 